\title{
Establishment and Development of the Disease Surveillance System
}

\author{
Ning Wen
}

\subsection{Basics of Disease Surveillance}

\subsubsection{Definition of Disease Surveillance}

Disease surveillance is the long-term, continuous, systematic collection of information about diseases and their relevant influencing factors, followed by analysis of the data in a timely manner to guide intervention measures and evaluate their effectiveness. This definition reflects the three basic characteristics of disease surveillance: (1) distributions and trends of diseases can be evaluated only through long-term, continuous, systematic collection of data; (2) original data can be translated to valuable information only through analysis and interpretation of the data; and (3) results can be fully utilized in the real world only after information is provided back to the relevant departments and personnel - hence the importance of timely feedback.

\subsubsection{Classification of Disease Surveillance}

\subsubsection{Passive Surveillance and Active Surveillance}

Passive surveillance is when lower level units routinely report surveillance data that the higher level units passively accept. In contrast, active surveillance involves higher level units conducting specialized investigations or requiring lower level

\footnotetext{
N. Wen $(\bowtie)$

National Immunization Program, Chinese Center for Disease Control and Prevention,

Beijing, China

e-mail: wenning@chinacdc.cn
} 
units to collect and report data in strict accordance with requirements. Notifiable infectious disease reporting that is used in most countries is passive surveillance. Special surveys, such as those conducted by CDCs in China to assess underreporting of infectious disease, and monitoring of certain diseases in accordance with standard requirements are examples of active surveillance. In general, active surveillance data quality is better than passive surveillance data quality.

\subsubsection{Routine Reporting and Sentinel Surveillance}

Routine reporting involves regular disease reports, which are used to understand the epidemiology of the disease. Routine surveillance collects data from all reporting resources (e.g., hospitals, institutions, or healthcare personnel). Examples are the notifiable infectious disease reporting system in all countries. The diseases covered vary by country. Sentinel surveillance refers to regular, quantitative monitoring of certain diseases in high-risk populations in well-described areas, depending on the epidemiological characteristics of the disease, with a purpose to understand more fully the epidemiology of the disease. Sentinel surveillance may require more time and resources than routine surveillance but generally provides more detailed information about the disease. An example is when nasopharyngeal swab samples are collected from each patient in selected areas where influenza sentinel surveillance sites are established to identify the type of influenza viruses circulating. The data collection method is not likely to be applicable to all patients but must be done systematically in sentinel surveillance.

\subsubsection{Surveillance Cases and Actual Cases}

Because there is no clear distinction between disease and health, when using clinical diagnostic criteria to detect cases, there will be a number of missed diagnoses and misdiagnoses. In public health surveillance, it is preferable to pay less attention to the accuracy of a diagnosis in an individual case but to emphasize unified, standard clinical diagnostic criteria with good potential for operationalization. Cases detected using the diagnostic criteria are called surveillance cases. The proportion of actual cases to surveillance cases should be as high as possible, and the relevant proportions and their variation should be estimated.

\subsection{China's Notifiable Infectious Disease System}

\subsubsection{Diseases Included in Notifiable Infectious Disease System}

The list of notifiable diseases differs in different countries. "Notifiable infectious disease" refers to an infectious disease that should be reported and for which patients should be treated and possibly isolated according to the law. Notifiable infectious diseases are often ones that spread fast and have severe outcomes and high fatality rates. According to the Chinese law, the notifiable infectious diseases in China are divided into categories $\mathrm{A}, \mathrm{B}$, and $\mathrm{C}$, with different reporting requirements for each 
category. There are 39 notifiable infectious diseases in China, including 2 category A diseases, 26 category B diseases, and 11 category $\mathrm{C}$ diseases.

\subsubsection{History of Notifiable Infectious Disease Surveillance in China}

Disease surveillance was initially established in the 1930s in China, when Keqian Chen conducted a small-scale epidemiological surveillance project in Ding county, Hebei province. After 1950, New China established an epidemic reporting system and began to monitor epidemics.

In June 1955, China MoH issued the first "Law on Infectious Disease Management" and established the national epidemic reporting system, in which the 18 notifiable infectious diseases were divided into category A or B. Three diseases belonged to category A: plague, cholera, and smallpox; 15 belonged to category B: Japanese encephalitis, diphtheria, typhus, relapsing fever, dysentery (bacterial and amebic), typhoid and paratyphoid, scarlet fever, meningococcal meningitis, measles, poliomyelitis, pertussis, anthrax, brucellosis, tick-borne encephalitis, and rabies.

In September 1978, there were 25 notifiable diseases, still divided into two categories. Starting September 1, 1989, the list of notifiable diseases was expanded to 35 diseases in three categories - A, B, and C. Two diseases were category A, plague and cholera; 22 were category B, viral hepatitis, bacillary and amebic dysentery, typhoid and paratyphoid, AIDS, gonorrhea, syphilis, poliomyelitis, measles, pertussis, diphtheria, meningococcal meningitis, scarlet fever, epidemic hemorrhagic fever, rabies, leptospirosis, brucellosis, anthrax, epidemic and endemic typhus, JE, malaria, dengue fever, and Kala-azar; and 11 were category C, tuberculosis, schistosomiasis, filariasis, echinococcosis, leprosy, influenza, mumps, measles, neonatal tetanus, acute hemorrhagic conjunctivitis, and infectious diarrhea other than cholera, dysentery, and typhoid and paratyphoid.

In December 2004, SARS and human infection from highly pathogenic avian influenza were included into category B reportable diseases, and some diseases were moved between categories B and C. In May 2008, hand, foot, and mouth disease was included into category C, and on April 30, 2009, influenza A/H1N1 (formerly called swine flu) was included into category B.

In October 2013, avian influenza H7N9 infection was included into category B; influenza $\mathrm{A} / \mathrm{H} 1 \mathrm{~N} 1$ was moved from category $\mathrm{B}$ to category $\mathrm{C}$ and was incorporated into the management system for seasonal influenza; human infection with highly pathogenic avian will no longer be prevented and controlled as category A.

\subsubsection{Noncommunicable Disease Surveillance}

With the changing spectrum of human morbidity and mortality, the diseases covered by surveillance were expanded to noninfectious diseases, such as malignant tumors, 
cardiovascular and cerebrovascular diseases, occupational diseases, birth defects, and other noncommunicable diseases.

\subsubsection{Other Public Health Surveillance}

In order to achieve specific public health goals, various surveillance activities are conducted, including environmental surveillance, nutrition surveillance, infant and maternal mortality surveillance, adverse drug reaction surveillance, family planning surveillance, and other conditions or events as needed to assess progress toward goals.

\subsection{Surveillance of Vaccine-Preventable Infectious Disease in China}

The ultimate goal of the immunization program is to control and eliminate infectious diseases.

In addition to routine surveillance of infectious diseases, some diseases targeted by the immunization program required specific surveillance. Poliomyelitis serves as a good example. The acute flaccid paralysis (AFP) surveillance system was established in 1991, in which all acute flaccid paralysis cases of unknown cause among children under 15 years old were identified and evaluated. The AFP surveillance system has played a critically important role in the polio eradication program.

\subsubsection{Acute Flaccid Paralysis (AFP) Surveillance System}

\subsubsection{Establishment of the AFP Surveillance System in China}

The AFP surveillance system was established in the context of polio eradication: The WHO region of the Americas (PAHO) adopted a goal in 1985 to eradicate wild poliovirus by 1990, and in 1988, the WHO Western Pacific Region (WPRO) adopted a goal to eradicate poliomyelitis by 1995 . As a member of WPRO, China committed to eradication of poliomyelitis in 1991. Jiangsu, Shandong, Henan, Hebei, and Anhui provinces set up AFP surveillance systems that year, and the system was called the polio special reporting system. $\mathrm{MoH}$ issued the "polio special reporting standard" in 1992, and the system was renamed as the AFP surveillance system in 1994. The polio special reporting system included a computerized database for AFP cases: a zero-case reporting database and a polio laboratory database at provincial Epidemic Prevention Stations (EPS, subsequently renamed to CDC). In 1993, except for Tibet, all provinces in China established AFP surveillance systems. AFP cases were reported by hospitals and by personnel at the county level CDC through active surveillance. Surveillance data were collected and summarized level by level. Since 1995, the AFP surveillance system has become better and better, and surveillance quality has been gradually improved. More than 5000 AFP cases have been 
reported annually with an AFP rate of more than 1/100,000 in China among children under 15 years old.

\subsubsection{Definition for AFP Surveillance}

The AFP surveillance system reports all cases with acute flaccid paralysis symptoms in children aged less than 15 years and all clinical polio cases regardless of age.

The clinical features of AFP are acute onset, decreased muscle tone, decreased muscle strength, and decreased or nonexistent tendon reflexes. Because several diseases have similar clinical features, AFP is monitored in order to detect possible polio cases.

The most common diseases diagnosed among AFP cases in China are (1) poliomyelitis; (2) Guillain-Barre syndrome (infectious polyradiculitis, GBS); (3) transverse myelitis, myelitis, encephalomyelitis, and nerve root acute myelitis; (4) polyneuropathy (multi polyneuropathy due to drug, toxic substances, and unexplained reasons); (5) nerve root inflammation; (6) traumatic neuritis (including neuritis due to injection in gluteal muscle); (7) single neuritis; (8) plexus neuritis; (9) periodic paralysis (including low potassium paralysis, high potassium paralysis, and normal potassium paralysis); (10) myopathy (including myasthenia gravis, poisoning, and unexplained myopathy); (11) acute polymyositis; (12) botulism; (13) quadriplegia, paraplegia, and monoplegia (unknown cause); and (14) transient limb paralysis.

\subsubsection{Change of Reporting Patterns}

The reporting pattern in China experienced four stages: in the first stage, paperbased reports were used; in the second stage, paper reports were augmented with computer-disk reporting; in the third stage (2004-2011), internet browser reporting was used; and in the fourth stage, beginning in 2012, direct reporting through the Internet was integrated into the notifiable infectious disease reporting system.

From 2004 to 2011, a client-server system was used for China's AFP surveillance. The "clients" were at provincial, prefecture, and county levels; they used software to download data from the national server and used analysis functions in the client software to identify and characterize AFP surveillance indicators. This client-server system made it easier to collect AFP surveillance data with automatic analyses, although some disadvantages continued to exist. First, the client software needed to be upgraded to keep pace with surveillance standards. However, each time the client software was upgraded or personal computers were upgraded or reinstalled, the client software need to also be reinstalled, making maintenance of client software burdensome. Second, AFP data need to be uploaded to the national server through the clients' software without real-time uploading and updating of the data between the client software and server.

To further improve the sensitivity of the surveillance system and learn the features of AFP cases in a timely manner, a direct reporting system through the Internet was implemented in 2012. It was called the "acute flaccid paralysis surveillance information report and management system" (hereinafter refer to real-time online 
AFP surveillance system). The system, which uses Internet browsers, is considered a real-time surveillance system. Data entry is performed through a personal computer using web browsers to log on to the national server. The system has been improved and upgraded continuously to meet the needs of AFP surveillance; however, most upgrades need to be done only on the server side. All case information is transferred to the server on a real-time basis. Persons with access authority can obtain access to the real-time data and can conduct analyses to determine results in real time. This system is divided into three modules: case reporting, case information management, and data analysis. Specific administrative rights are allocated to staff commensurate with their roles in AFP surveillance.

The process of AFP reporting and management is as follows. Medical institutions and county CDCs with reporting permission report AFP cases by completing an "infectious disease reporting card" in the "real-time online AFP surveillance system." Epidemiologists at county CDCs conduct epidemiological investigations of AFP cases after receiving AFP reports; they collect stool samples during this time. The CDC with proper authority enters data in the "real-time online AFP surveillance system," including epidemiological information and stool sample collection dates. Stool samples are sent by county CDCs to province CDC laboratories, and staff at provincial laboratories enter the "real-time online AFP surveillance system" to indicate receipt of stools and results of laboratory tests when they are available. If the laboratory result is positive for poliomyelitis, samples are sent to the national laboratory for detailed characterization. Provincial laboratories enter the "real-time online AFP surveillance system" to complete the feedback loop from the national laboratory. County CDCs conduct follow-up investigations of AFP cases and enter investigation information into the system. A provincial expert group makes the final diagnosis and classification of AFP cases to determine whether polio is excluded or not. After final diagnosis and classification, staff at provincial CDC enter the final diagnostic classification into the "real-time online AFP surveillance system" (Fig. 3.1).

The polio laboratory network is an integral component of AFP surveillance. Established in 1992, China's polio laboratory network consists of polio laboratories at China CDC (certificated as Western Pacific Region's reference laboratory by the WHO in 1995) and 31 provincial CDCs.

Cell culture, virus isolation, and serological identification are performed at the provincial polio laboratories using reagents, cell lines, and procedures recommended by the WHO. All polioviruses isolated by provincial polio laboratories are sent to the national polio laboratory for type identification, sequencing, and other analyses. The responsibilities of national polio reference laboratory include type identification and distinguishing vaccine strains from vaccine-derived polioviruses (VDPVs) and wild polioviruses. Differences from vaccine strains are determined using polymerase chain reaction - restriction fragment length polymorphism (PCRRFLP) or enzyme-linked immunosorbent assay (ELISA).

The national polio reference laboratory is one of the three WHO-certified reference laboratories in the Western Pacific Region. In accordance with WHO certification standards, China's provincial polio laboratories have also been certificated as national level polio laboratories. 


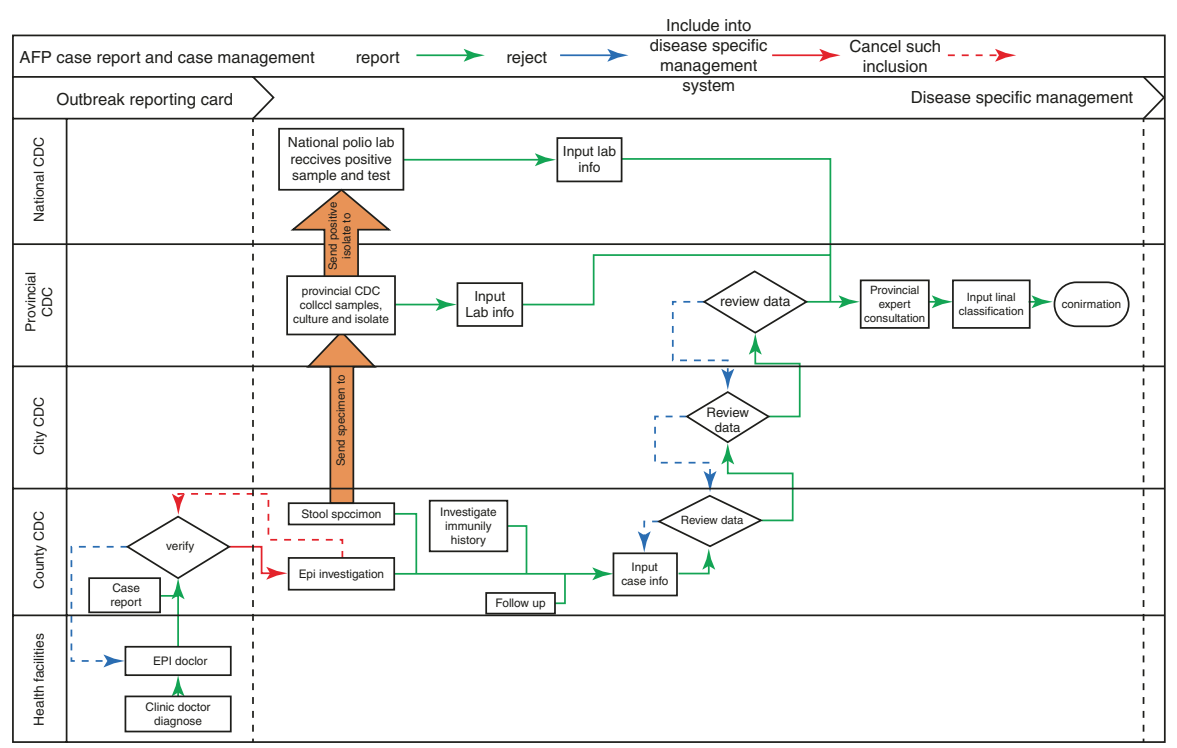

Fig. 3.1 Procedure of the Real-time Online AFP Surveillance System

As a reference laboratory in the Western Pacific Region, on-site certification and performance assessment of China's national polio laboratory has been conducted by WHO experts every year since 1996. On-site certification and performance assessment of provincial polio laboratories are conducted every 1-3 years, depending on the laboratory's most recent assessment result.

\subsubsection{Other Disease-Specific Surveillance Systems}

In addition to the AFP surveillance system, there are three other surveillance systems: measles, meningococcal meningitis, and Japanese encephalitis.

Measles surveillance data aggregated by province has been reported through the national notifiable disease reporting system (NNDRS) since 1959. In 1998 a standard NNDRS-based measles surveillance program was developed. The current realtime dynamic measles surveillance system (MSS) combined epidemiological and laboratory surveillance. From 2004, each province began to report measles cases through the "China Immunization Program and Surveillance Information Management System.”

China established JE surveillance in 2007 and meningococcal meningitis in 2008. Surveillance systems play an important role in the control of diseases. 